\section{IGCP Projects 2009}

(OET - on extended term)

No.480 Tectonics of Central Asia

Project leaders: B. Natal'in (Turkey), A. Yin (United States), A.M.C. Sengör (Turkey),

M. Kuzmin (Russia), Shuwen Dong (China)

Duration: 2005-2009

Website: http://www.igcp.itu.edu.tr/

No.493 The Rise and Fall of the Vendian Biota

Project leaders: M. Fedonkin (Russia), P. Vickers-Rich (Australia), J. Gehling (Australia)

Duration: 2003-2007 (OET)

Website: http://www.geosci.monash.edu.au/ precsite

\section{No.495 Quaternary Land-Ocean} Interactions

Project leaders: A. Long (United Kingdom), S. Islam (Bangladesh)

Duration: 2004-2008 (OET)

Website: www.geography.dur.ac.uk/projects/ igcp 495

\section{No.497 The Rheic Ocean}

Project leaders: U. Linnemann (Germany), R. D. Nance (United States), M. de Wit (South Africa), E. Bozkurt (Turkey), P. Kraft (Czech Republic), F. Pereira (Portugal), R. A. Strachan (United Kingdom)

Duration: 2004-2008 (OET)

Website: http://www.snsd.de/igcp497/

No.499 Devonian land-sea interaction:

Evolution of Ecosystems and Climate in the

Devonian

P. Königshof (Germany), J. Lazauskiene (Lithuania), E. Schindler (Germany), Volker Wilde (Germany) and N. Yalçin (Turkey)

Duration: 2004-2008 (OET)

Website: http://www.senckenberg.de/igcp499

No. 500 Dryland Change: Past, Present, Future

Project leader: D.Thomas (United Kingdom) Duration: 2004-2008 (OET)

Website: http://igcp500.ouce.ox.ac.uk/

No.502 Global Comparison of Volcanichosted Massive Sulphide Districts

Project leaders: R.Allen (Sweden), F. Tornos (Spain), J. Peter (Canada), N. Çagatay (Turkey)

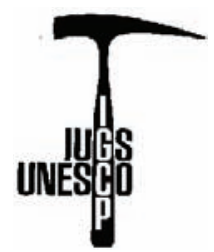

Duration: 2004-2008 (OET)

Website: http://www.1tu.se/tkg/ http://www.1tu.se/tkg/avd/kgo/forsk/IGCP

No.503 Ordovician Palaeogeography and Palaeoclimate

Project leaders: T. Servais (France), D.A.T. Harper (Denmark), J. Li (China), A. Munnecke (Germany), A. W. Owen (United Kingdom), P.M. Sheehan (United States)

Duration: 2004-2008 (OET)

Website: http://sarv.gi.ee/igcp503/

No.506 Marine and Non-marine Jurassic Project leaders: Jingeng Sha (China), Nicol Morton (France), W. A.P. Wimbledon (United Kingdom), Paul E. Olsen (United States), Alberto G. Riccardi (Argentina), Grzegorz (Gregory) Pieñkowski (Poland), Yongdong Wang (China)

Duration: 2005-2009

Website: http://www.nigpas.ac.cn/ IGCP506index.asp

No.507 Paleoclimates of the Cretaceous in Asia

Project leaders: Yong Il Lee (Korea), Xiaoqiao Wan (China), Takashi Sakai (Japan), and Krishnan Ayyasami (India)

Duration: 2006-2010

Website: http://igcp507.kopri.re.kr/

No.509 Palaeoproterozoic Supercontinents and Global Evolution

Project leaders: S.M. Reddy (Australia), D. Evans (United States), R. Mazumder (India)

Duration: 2005-2009

Website: http://earth.geology.yale.edu/ igcp509/

No.510 A-type Granites and Related Rock through Time

Project leaders: Roberto Dall'Agnol (Brazil), Carol D. Frost (United States), O. Tapani Rämö (Finland), L.J. Robb (South Africa) Duration: 2005-2009

Website: http://www.igcp-510.org

No.511 Submarine Mass Movements and Their Consequences

Project leaders: Jacques Locat (Canada), Juergen Mienert (Norway) and Roger Urgeles (Spain)- (IOC link)

Duration: 2005-2009

Website: http://www.geohazards.no/IGCP511
No.512 Neoproterozoic Ice Ages

Project leaders: Graham A. Shields (Australia), Emmanuelle Arnaud (Canada)

Duration: 2005-2009

Website: www.IGCP512.org

\section{No.513 Karst Aquifers and Water}

Resources

Project leaders: Chris Groves (United States), Yuan Daoxian (China), Bartolome Andreo-Novarro (Spain), Heather Viles (United Kingdom)

Duration: 2005-2009

Website: http://hoffman.wku.edu/igcp/ 513.html (General information) http://hoffman.wku.edu/karst2007/ k2007.html http://www.wku.edu/cehp http://www.cosis.net/members/meetings/ sessions/information.php?p_id=247\& s_id=4433

No.514 Fluvial Palaeosystems: Evolution and Mineral Deposits

Project leaders: A. Duk-Rodkin (Canada), Baohong Hou (Australia), Li Ziying (China), Vladimir Dolgopolov (Kazakhstan), N. Patyk-Kara (Russia) passed away in 2008

Duration: 2005-2009

Website: http://www.igem.ru/igcp514/

No.516 Geological Anatomy of East and South East Asia

Project leaders: Ken-ichiro Hisada (Japan), Punya Charusiri (Thailand), Byung-Joo Lee (Rep. of Korea), Xiaochi Jin (China)

Duration: 2005-2009

Website: http://staff.aist.go.jp/hara-hide/ igcp516

No.519 Hydrogeology, Hydrochemistry and Management of Coastal Aquifers on the Atlantic Coast of South America

Project leaders: Emilia Bocanegra (Argentina), Emilio Custodio (Spain), Marisol Manzano (Spain), Gerson Cardoso (Brazil), Jenny Reynolds Vargas (Costa Rica),

Duration: 2005-2009

Website: http://www.mdp.edu.ar/exactas/ geologia/cgcyc/hidrogeologia.html

No.521 Black sea Mediterranean Corridor during the last $30 \mathrm{ky}$ : Sea level change and human adaptation

Project leaders: Valentina Yanko-Hombach (Canada), Yucel Yilmaz (Turkey), Pavel Dolukhanov (United Kingdom)

Duration: 2005-2009

Website: http://www.avalon-institute.org/ IGCP

http://black.sealevel.ca 
http://www.bridge.bris.ac.uk/projects/ EMBSECBIO

http://www.paleontol.geo.sfedu.ru

\section{No.523 GROWNET - Gobal Ground Water Network \\ Project leaders: Shrikant Daji Limaye (India), Antony J Reedman (United Kingdom) \\ Duration: 2005-2009 \\ Website: http://www.igcp-grownet.org}

No.524 Arc-Continent Collision

Project leaders: Denis Brown (Spain), ChiYue Huang (Taiwan)

Duration: 2007-2009

www.ija.csic.es/gt/IGCP524

No.526 Risks Resources and Record of the Past on the Continental Shelf

Project leaders: Francesco L.atino Chiocci (Italy), Lindsay Collins (Australia), Michel Michaelovitch de Mahiques (Brazil), Renée Hetherington (Canada)

Duration: 2007(-2011).

No.529 Availability of groundwater resources in selected urban areas in Southern African Development Community (SADC) region

Project leader: Imasiku A. Nyambe (Zambia) Duration: 2007-2011

No.540 Gold-bearing hydrothermal fluids of oregenic deposits

Project leaders: P.S. Garofalo (Italy), J.R. Ridley (USA), Vsevolod Prokof'ev (Russia)

Duration: 2007-2011

Website: http://www.geomin.unibo.it/ igcp_540

No.543 Low-temperature thermochronology: applications and interlaboratory calibration

Project leaders: Massimiliano Zattin (Italy), J. I. Garver (USA), Vitaliy A. Privalov (Ukraine), Alexei V. Soloviev (Russia), Cornelia Spiegel (Germany), Maarten de Wit (South Africa), Dewen Zheng (China) Duration: 2007-2010

No.546 Subduction zones of the Caribbean

Project leaders: Antonio Garcia-Casco (Spain), Uwe Martens (USA)

Duration: 2007-2011

Website: http://www.ugr.es/ agcasco/ igcp546/
No.555 Rapid Environmental/Climate Change in the Cretaceous Greenhouse World

Project leaders: Chengshan Wang (China), Robert Scott (USA), Hugh Jenkyns (UK), Michael Wagreich (Austria), William Hay (USA); Zakharov Y.D. (Russia)

Duration: 2007-2010

Website: www.cretaceousworld.com/igcp 555

No.557 Diamonds, xenoliths and kimberlites

Project leaders: Holger Sommer (Botswana), Klaus Regenauer-Lieb (Australia), Christoph Hauzenberger (Austria) Jonathan Kashabano (Tanzania), Gétan Moloto-A-Kenguemba (Central African Republic)

Duration: 2007-2011

Website: http://igcp557.uni-graz.at/

No.559 Crustal Architecture and landscape Evolution

Project leaders: Bruce R. Goleby (Australia) and 14 members (USA, Canada, China, Finland, Netherlands, New Zealand, Russia)

Duration: 2008-2012

No.565 Geodetic Monitoring of the Global Water Cycle

Project leaders: Hans-Peter Plag (USA), Richard S. Gross (USA), Markus Rothacher (Germany), Norman L. Miller (USA), Susanna Zerbini (Italy), Chris Rizos (Australia)

Duration: 2008-2012

No.567 Earthquake Archaeology Archaeoseismology along the AlpineHimalayan seismic zone

Project leaders: Manuel Sintubin (Belgium), Iain Stewart (United Kingdom), Tina Niemi (USA), Erhan Altunel (Turkey)

Duration: 2008-2012

No.571 Radon, health and natural hazards

Project leaders: Gavin K. Gillmore (UK), Robin G.M. Crockett (UK), Frederic Perrier (France), Tadeusz Przylibski (Poland), Vivek Walia (Taiwan of China), Bikram Jit Singh Bajwa (India)

Duration: 2009-2014

No.572 Permian-Triassic ecosystems

Project leaders: Zhong Qiang Chen (Australia), Richard J. Twitchett (United Kingdom), Jinnan Tong (China), Margret
L. Fraiser (USA), Sylvie Crasquin (France), Steve Kershaw (United Kingdom), Thomas J. Algeo (USA), Kliti Grice (Australia)

Duration: 2008-2012

No.574 Bending and Bent Orogens, and Continental Ribbons

Project leaders: Stephen T. Johnston, (Canada), Gabriel Gutierrez-Alonso (Spain), Arlo Weil (USA)

Duration: 2009-2014

No.580 Application of magnetic suscepti-bility on Paleozoic sedimentary rocks

Project leaders: Anne-Christine da Silva (Belgium), Michael T. Whalen (USA), Jindrich Hladil (Czech Republic), Daizhao Chen (China), Simo Spassov (Belgium), Frederic Boulvain (Belgium), Prof. Devleeschouwer Xavier (Belgium)

Duration: 2009-2014

\section{No.581 Evolution of Asian River} Systems

Project leaders: Hongbo Zheng (China), Ryuji Tada (Japan), Peter Clift (UK), Masood Ahmad (India), Zheng-Xiang Li (Australia), Kuo-Yen Wei (Taiwan of China)

Duration: 2009-2014

No.582 Tropical Rivers: Hydro-Physical Processes, Impacts, Hazards and Management

Project leaders: Edgardo M. Latrubesse (Argentina), Rajiv Sinha (India), Jose C. Stevaux (Brazil)

Duration: 2009-2014

Funded projects

O.E.T.

Total 37

\section{IGCP Secretariat}

UNESCO, Division of Ecological and Earth Sciences

1, Rue Miollis

75732 Paris Cedex 15

France

Tel: +33145684118

Fax: +33145685822

www.unesco.org/sciencelearth

E-mail:igcp@unesco.org 